\title{
General Solution of Laplace and Poisson Equations in a Multiple-Connected Circular Domain: Applications to Torsion
}

\author{
L.N. Epele, H. Fanchiotti, C.A. García Canal \\ Instituto de Física La Plata, CCT La Plata, CONICET and Laboratorio de Física Teórica \\ Departamento de Física, Facultad de Ciencias Exactas, Universidad Nacional de La Plata \\ CC 67, 1900 La Plata, Argentina
}

March 1, 2012

\begin{abstract}
The general solution of Laplace and Poisson equations in multiple-connected domains, in terms of the group of automorphism, is summarized. The particular application to the analysis of rods under torsion is presented. Different generalizations are discussed.
\end{abstract}

\section{Introduction}

The study of the behavior of rods under torsion is fundamental in the design of structural elements. For this reason it is one of the classical problem in the theory of elasticity [1], [2], [3]. At present, there are some analytical solutions found for simple shapes as those defined by circles, annulus, ellipses or other elementary geometrical forms. In any other cases, numerical methods as the finite element one, are necessary[5].

At his respect, it is worth notice that a wide variety of technological problems related not only to torsion of bars, but also in connection with electric power transmission, the heat transport etc. can be reduced to the determination of harmonic functions and Green functions in a multiply connected domain. In fact, the cylindrical symmetry present in a large variety of cases, allows one to restrict the original problem to the solution of the Laplace, or the Poisson equation, in a two dimensional domain with holes. In general, the boundaries could be of any geometrical form, making the exact solution of the problem quite difficult or even impossible.

We are particularly interested in the solution of the Laplace and the Poisson equations in the presence of a system of hollowed tubes with circular section and Dirichlet boundary conditions: the unknown function (namely the stress function, the temperature, the electric potential, etc) is given on the boundaries defined by the holes. In recent years, this problem has received considerable attention in engineering literature, where various approximate methods of varying degrees of accuracy have been devised in order to solve it [4], [5]. However, the construction of harmonic functions in multiply connected domains is well known and extensive mathematical literature exists on the subject [6]. The general results that we shall summarize here are already indicated in a nice mathematical paper published by Burnside in 1891 [7]. This kind of problems can be exactly solved, even in the case in which the holes are not circular but have any shape, in terms of the so-called "automorphic functions" which are a generalization for surfaces with many holes of the elliptic functions, solutions of the harmonic problems in surfaces with only one hole. These exact analytic solutions are given in terms of series called "Poincare theta series". It is worth mentioning that there are recent contributions on constructive theory of functions in multiply connected domains designed to cope with a variety of problems, most of them related to fluid dynamics, mainly developed by D.G. Crowdy and collaborators [8].

The main purpose of this paper is to recall this general method of solution based on the group of automorphisms of the domain defined by the section of the structure under study. In a 1974 paper [4] we 
have already presented the exact solution of the electrostatic problem for a system of parallel cylindrical conductors based on the mentioned method. The idea of the present paper is to translate all the procedure to the problem of elasticity. In particular, to the analysis of rods under torsion. In this case one needs to deal explicitly with Green functions, because the equation of the stress function is not homogeneous. We show that these exact solutions are easy to handle in practical technological applications related to the analysis of torsion of bars.

We present in the next Section 2, in a simplify way, the mathematical background that is necessary for dealing with the general solution of Laplace and Poisson equations in a multiply connected domain. Section 3 is devoted to the presentation and solution of a simple case of interest in the theory of elasticity, to be taken as an example. Finally in Section 4 we state our conclusions.

\section{Mathematical Background}

\subsection{Projective Transformations}

The exact solution of two-dimensional harmonic problems in multiply connected domains, the basis of our problems of interest, rests heavily on the systematic use of projective transformations. We shall therefore present here a summary of the elementary properties of these transformations that are used in the rest of the paper.

A projective transformation is a conformal transformation of the form

$$
z^{\prime}=T(z)=\frac{(a z+b)}{(c z+d)}
$$

These transformations are the only conformal transformations that map the whole of the complex plane onto itself. They also map circles or straight lines into circles or straight lines. The parameters $a, b, c, d$ are in general complex. Notice however that the transformation (1) depends only on three of them because, being a homogeneous transformation, it does not change if we multiply all parameters by the same complex constant. We can therefore always choose one of them to be equal to 1 or alternatively, as we shall do in what follows, we can impose the normalization condition $a d-b c=1$. Another important point to be noticed is that since we have three parameters at our disposal, we can always find a projective transformation that maps three given points into three other given points in the complex plane.

It is very convenient to associate to the projective transformation $T(z)$ a $2 \times 2$ matrix

$$
\tau=\left(\begin{array}{ll}
a & b \\
c & d
\end{array}\right)
$$

with $\operatorname{det} \tau=a d-b c=1$. This matrix arises very naturally if one introduces homogeneous coordinates in (1) and writes $z=x / y ; z^{\prime}=x^{\prime} / y^{\prime}$, all of them being complex numbers. Then it follows that (1) can be written in matrix notation as

$$
\left(\begin{array}{l}
x^{\prime} \\
y^{\prime}
\end{array}\right)=\left(\begin{array}{ll}
a & b \\
c & d
\end{array}\right)\left(\begin{array}{l}
x \\
y
\end{array}\right) .
$$

Using this representation it can now be easily checked that if we perform two successive projective transformations

$$
z^{\prime \prime}=T^{\prime \prime}(z)=T^{\prime}(T(z))=\frac{\left(a^{\prime \prime} z+b^{\prime \prime}\right)}{\left(c^{\prime \prime} z+d^{\prime \prime}\right)}
$$

then the parameters of the resulting projective transformation $T^{\prime \prime}(z)$ are given by a $2 \times 2$ matrix equal to the product $\tau^{\prime \prime}=\tau^{\prime} \tau$. Notice that $\tau^{\prime \prime}$ is automatically normalized because of the properties of the determinants. Therefore, the operation of performing successive projective transformations is entirely equivalent to the matrix multiplication of the associated matrices. One therefore usually refers to the projective transformation $T^{\prime \prime}$ as the product of the transformations $T^{\prime} T$. 
We shall need very often the concepts of "invariant points" and "multiplier" of a given projective transformation $T(z)$. The equation

$$
T(z)=z
$$

has in general two distinct complex roots that we shall denote by $\xi$ and $\eta$ and which are called the invariant points of $T$. It may happen that $\xi=\eta$ in which case the transformation $T(z)$ is called parabolic, but we shall never need to consider parabolic transformations in what follows, so from now on we shall assume that the roots are different.

The invariant points are easily obtained from the representation of $T(z)$ in terms of homogeneous coordinates. Notice that the couples $(x, y)$ and $(\lambda x, \lambda y)$ give the same complex number $z=x / y$. Let us now compute the eigenvalue and the eigenvectors of $\tau$. We shall find in general two complex eigenvalues $\lambda_{1,2}$ and the associated complex eigenvectors $\left(x_{1}, y_{1}\right)$ and $\left(x_{2}, y_{2}\right)$, respectively. So we conclude that the eigenvectors of $\tau$ are the homogeneous coordinate representation of the invariant points. Then we find

$$
\xi=\frac{x_{1}}{y_{1}} ; \eta=\frac{x_{2}}{y_{2}}
$$

We finally define the multiplier $\kappa$ as the ratio of the two eigenvalues of $\tau$

$$
\kappa=\frac{\lambda_{1}}{\lambda_{2}}
$$

so the eigenvectors and eigenvalues of $\tau$ determine the invariant points and the multiplier of $T(z)$ respectively. One can now use the three complex parameters $\xi, \eta$ and $\kappa$ to characterize the projective transformation $T(z)$, that in terms of these parameters can be written as

$$
\frac{T(z)-\xi}{T(z)-\eta}=\kappa \frac{z-\xi}{z-\eta}
$$

The parameter $\kappa$ is called multiplier because one can always perform a particular projective transformation that maps $(\xi, \eta)$ to $(0, \infty)$ as follows

$$
z^{\prime}=Q(z)=\frac{z-\xi}{z-\eta}
$$

Consequently, the projective transformation changes according to $T^{\prime}=Q T Q^{-1}$ In terms of the transformed variable, (8) reads

$$
T^{\prime}\left(z^{\prime}\right)=\kappa z^{\prime}
$$

and the projective transformation $T^{\prime}\left(z^{\prime}\right)$ reduces to a scale transformation in the $z^{\prime}$ complex plane. We use below this property to relate two geometrical situations of interest. Namely, the case of a circle and a straight line and the case of two coaxial cylinders.

An important point to be emphasized here is that one can always choose the ratio of the eigenvalues $\lambda_{1} / \lambda_{2}$ in such a way that $|\kappa|<1$. So we define $\xi$ as the invariant point determined from the eigenvector of $\tau$ associated with the smallest eigenvalue (in magnitude).

Another useful concept that we need in what follows is that of "isometric circle" of a transformation $T(z)$ (and that of its inverse transformation $T^{-1}(z)$ ). Given $T(z)$, its inverse transformation has an associated matrix $\tau^{-1}$, and is given by

$$
T^{-1}(z)=\frac{(d z+b)}{(-c z+a)} ; d a+b c=1
$$

The isometric circle of $T(z)$ - denoted by $I(T)$ - is the circle defined by

$$
|c z+d|=1
$$

so its center $J$ and radius $R$ are given by

$$
R=\frac{1}{|c|} ; J=-\frac{d}{c}
$$


Likewise the isometric circle of $T^{-1}(z)$ denoted by $I\left(T^{-1}\right)$ and defined by $|-c z+a|=1$ has

$$
R=\frac{1}{|c|} ; \quad J^{-1}=\frac{a}{c}
$$

These concepts are very important because the radii and centers of the isometric circles of some projective transformations will be identified with the radii and the centers of the cylindrical boundaries we are interested in. Moreover, the fundamental result that we shall need in what follows is that $T(z)$ maps its isometric circle $I(T)$ into $I\left(T^{-1}\right)$ and the interior (or exterior) of $I(T)$ into the exterior (or interior) of $I\left(T^{-1}\right)$. Likewise, $T^{-1}(z)$ maps $I\left(T^{-1}\right)$ into $I(T)$ and the interior (or exterior) of $I\left(T^{-1}\right)$ into the exterior (or interior) of $I(T)$.

Since a circle is uniquely determined by three points in the complex plane, and projective transformations transform circles into circles and are also uniquely determined by stating how three given points in the complex plane transform into three other given points, it is clear that one can always find a projective transformation $T$ that maps a given circle $C$ into another given circle $C^{\prime}$. However, if $C$ and $C^{\prime}$ have the same radius, the problem of finding $T$ is particularly simple, because $C$ will be the isometric circle of $T$, and $C^{\prime}$ the isometric circle of $T^{-1}$. The formulae written above allow us to find the parameters of $T$ in terms of the radius $R$ and centers of the circles. We shall come back to this point when presenting the example in a next section.

We shall need often to compute a power of a projective transformation, $T^{n}(z)$, defined as the successive application of $T(z), \mathrm{n}$ times. Clearly its associated matrix is $\tau^{n}$, and the eigenvectors of $\tau^{n}$ are the same as those of $\tau$. Therefore, the invariant points of $T^{n}(z)$ are the same as those of $T(z)$. It follows that the multiplier $\kappa_{n}$ of $T^{n}(z)$ is given by $\kappa_{n}=\kappa^{n}, \kappa$ being the multiplier of $T(z)$. This makes it simple therefore to compute the powers of a projective transformation. If the invariant points of $T(z)$ are mapped to $(0, \infty)$ by the transformation $z^{\prime}=S(z)$, then

$$
T^{n}\left(z^{\prime}\right)=\kappa^{n} z^{\prime}
$$

Consequently, from (8)

$$
\frac{T^{n}(z)-\xi}{T^{n}(z)-\eta}=\kappa^{n} \frac{z-\xi}{z-\eta}
$$

Since $|\kappa|<1$, when $n \rightarrow \infty, \kappa^{n} \rightarrow 0$ and we see immediately that

$$
\lim _{n \rightarrow \infty} T^{n}(z)=\xi
$$

This important result means that after a sufficient large number of transformations $T$, any point in the complex plane is mapped into a point arbitrarily close to the invariant point $\xi$ of $T$. One can rephrase this result by saying that the successive images of a point $z$ cluster at the invariant point $\xi$, where by image of $z$ we understand $T(z)$. Moreover, one can easily show that $T^{-1}(z)$ has the same multiplier $\kappa$ as $T(z)$, but the invariant points are interchanged: $\xi^{\prime}=\eta$ and $\eta^{\prime}=\xi$. Then we can also conclude that

$$
\lim _{n \rightarrow \infty} T^{-n}(z)=\eta
$$

\subsection{General Solution of Laplace Equation}

Just to make as clear as possible our presentation, let us start by considering the case of a system of $N$ cylindrical tubes placed above a plane. The plane is taken to be the real axis and the circular holes representing the cylinders are taken in the upper half-plane. We shall restrict ourselves to this configuration since the case of $N$ cylindrical tubes placed inside another cylinder can be transformed into the first case by means of a projective transformation. This fact is explicitly used in the analysis of torsion of bars that we present below. In the case presently under consideration one is interested in solving a two-dimensional Dirichlet problem in a multiply connected domain with $N+1$ boundaries $-N$ circles and the real axis - in which the unknown function $\phi(x, y)$ takes a constant value $\phi_{i}$ over the boundary and 0 on the plane. In this case, it is very convenient to introduce a set of $N+1$ harmonic functions called "harmonic measures" that are solutions of the Laplace equation and each one with the following boundary condition: the $i$ th harmonic 
measure $\omega_{i}$ takes the value 1 on the $i$ th boundary and zero on the others. Each tube and the plane have their associated harmonic measure. The harmonic measures can be calculated once the Green function of the system is known, but we shall obtain them in a different way. Once the harmonic measures $\omega_{i}(z)$ are known, the solution of the Dirichlet problem in which $\phi(z)$ takes the value $\phi_{i}$ on the $i$ th boundary is given by

$$
\phi(z)=\sum_{i=1}^{N+1} \phi_{i} \omega_{i}(z)
$$

as it can easily be checked by inspection. However, not all $N+1$ harmonic measures can be independent of each other. This can be seen considering the solution of the Dirichlet problem in which all $\phi_{i}=1$. Since a harmonic function cannot have local maxima or minima, the solution is a constant equal to 1 . Then

$$
\phi(z)=\sum_{i=1}^{N+1} \omega_{i}(z)=1
$$

We conclude then that there are only $N$ independent harmonic measures that we shall take as being the ones associated with the $N$ cylindrical boundaries. Using the last expression to eliminate $\omega_{N+1}$-the harmonic measure of the plane-we find

$$
\phi(z)=\phi_{N+1}+\sum_{i=1}^{N}\left(\phi_{i}-\phi_{N+1}\right) \omega_{i}(z)
$$

so we see that the only relevant physical quantities of interest for the determination of the distribution we are looking for, are the values the function takes measured with respect to the plane, as it was to be expected. As we already stated, without loss of generality we shall set $\phi_{N+1}=0$ in what follows.

The second step is to construct a set of $N$ basic projective transformations $T_{i} ;(i=1, \cdots, N)$ defined as follows: We call $C_{i}$ the original circles representing the cylindrical tubes and $C_{i}^{\prime}$ their images with respect to the plane (real axis). The parameters of the projective transformation $T_{i}$ are entirely determined in terms of the center and radius of the circle $C_{i}$. Remember that from

$$
T_{i}(z)=\frac{\left(a_{i} z+b_{i}\right)}{\left(c_{i} z+d_{i}\right)}
$$

one obtains that

$$
R_{i}=\left|c_{i}\right|^{-1}
$$

and

$$
J_{i}=-\frac{d_{i}}{c_{i}}=i R_{i} d_{i}
$$

The basic $T_{i}$ 's are called "generators". The identity and the products of any number of them and of their inverses give rise to an infinite set of projective transformations that form a group $T_{\alpha}$ called the "group of automorphism" of the surface with $N$ holes under consideration and is completely determined by the geometry of the problem.

Now, after the construction of the group of automorphism of the surface, we can introduce a set of analytic functions that are the building blocks of the harmonic measures. The basic object is the Poincaré theta series defined as

$$
\theta(z, a)=\sum_{\alpha} \frac{1}{\left(c_{\alpha} z+d_{\alpha}\right)^{2}} \frac{1}{\left(T_{\alpha}(z)-a_{\alpha}\right)}
$$

where the summation extends to all the elements of the group of automorphism including the identity $I$. The proof of the convergence of the Poincare theta series is one of the basic results in Burnside's paper. The key convergence factor is $\left(c_{\alpha} z+d_{\alpha}\right)^{-2}$, that occurs in every term of the series. This factor is always smaller in modulus than 1 inside (or outside) the corresponding isometric circle. Now a fundamental result concerning the group of automorphism states that the isometric circle of $T_{\alpha}$ is always inside the isometric 
circle of one of the generators, so we are sure that in the region of interest (outside the $2 N$ circles $C_{i}$ and $C_{i}^{\prime}$ ) this convergence factor is smaller than 1.

In terms of this Poincaré theta series, Burnside introduces $N$ functions $\theta\left(z, J_{i}\right)(i=1, \cdots, N)$, where $J_{i}$ is the center of the circle $C_{i}$ (the isometric circle of $T_{i}$ ). The integrals of these functions

$$
\phi_{i}(z)-\phi_{i}\left(z_{0}\right)=\int_{z_{0}}^{z} \theta\left(z, J_{i}\right) d z=\sum_{\alpha} \ln \left(\frac{T_{\alpha}(z)-J_{i}}{T_{\alpha}\left(z_{0}\right)-J_{i}}\right)
$$

form a basic set of $N$ analytic functions in terms of which the harmonic measures can be constructed. In this equation, $z_{0}$ stands for an arbitrary point of the complex plane where $\phi_{i}(z)$ is normalized to zero. By construction, these functions, called automorphic functions, satisfy the central property

$$
\phi_{i}\left(T_{\alpha}(z)\right)=\phi_{i}(z)
$$

for any element $T_{\alpha}$ of the group of automorphisms. These $N$ analytic functions $\phi_{i}(z)$ themselves have a nice physical interpretation which follows from three basic properties shown by Burnside:

- (i) The functions $\phi_{i}(z)$ are analytic functions of $z$ for any value of $z$ in the region outside the $2 N$ holes. Indeed, one can write $\phi_{i}(z)$ in the form

$$
\phi_{i}(z)=\ln \left(z-J_{i}\right)+\sum_{\alpha \neq I} \ln \left(\frac{z-J_{i \alpha}}{z-J_{\alpha}}\right)+\text { const. }
$$

This means that in the case of an electrostatic problem, of more intuitive interpretation, $\operatorname{Re} \phi_{i}(z)$ is precisely the potential distribution generated by an infinite set of sources located at the centers of isometric circles. Since we have already mentioned that the centers of the isometric circles of any element of the group are always inside some of the $2 \mathrm{~N}$ circles or is analytic in the region outside the cylindrical conductors.

- (ii) If we write the two harmonic functions associated with $\phi_{i}(z)=u_{i}(z)+i v_{i}(z) ; i=1 \cdots N$, then the functions $u_{i}(x, y)=\operatorname{Re} \phi_{i}(z)$ are constant on the $2 N$ circular boundaries and on the real axis. This means that they are solutions of $N$ Dirichlet problems in which the unknown takes some (yet to be determined) constant values on the boundaries and on the real axis.

- (iii) $u_{i}(x, y)$ are single valued in the domain under consideration, but the conjugate functions $v_{i}(x, y)$ are not.

We have already remarked that the functions $u_{i}(x, y)$ are the solutions of Dirichlet problems in which the circular boundaries and the real axis are at constant values of the function under analysis. Let us now consider the flow $q$ generated by the function $u_{i}(x, y)$. We can compute the total flow per unit length going out from the $j$ th cylindrical tube. Using the Cauchy-Riemann relations, we find

$$
q_{j}=\oint_{\Gamma_{j}} \frac{\partial u_{i}(x, y)}{\partial n} d s=\oint_{\Gamma_{j}} \frac{\partial v_{i}(x, y)}{\partial s} d s=2 \pi \delta_{i j}
$$

so we conclude that the harmonic functions $u_{i}(x, y)$ give the distribution corresponding to a physical situation where the boundary $C_{i}$ gives rise to a flow per unit length $q_{i}=2 \pi$, and all the other boundaries have zero net flow per unit length.

Let us go back for a moment to (22) and examine more closely the structure of the function whose real part as we have just seen represents the distribution generated by the system of $N$ cylindrical tubes kept at some constant (but yet unknown) values but with a net flow per unit length equal to 1 inside the $i$ th tube and zero on the others.

Finally, we come to the point of determining the boundary values of the set of $u_{i}(x, y)$. First note that since they are defined up to an arbitrary constant, we can always choose this constant so as to make them 
vanish on the real axis. Going back to (20), this is accomplished by choosing $\phi_{i}\left(z_{0}\right)=0$ and the normalization point $z_{0}$ somewhere on the real axis.

Let us now consider a point $A_{j}$ on the $j$ th circular boundary $C_{j}$. We know by the definition of the projective transformation $T_{j}$ that the point $A_{j}^{\prime}=T_{j}\left(A_{j}\right)$ is on the image boundary $C_{j}^{\prime}$. We can now compute how much the analytic function $\phi_{i}(z)$ changes when going from the boundary $C_{j}$ to its image $C_{j}^{\prime}$ and define a matrix by

$$
A_{i j}=\phi_{i}\left(T_{j}\left(A_{j}\right)\right)-\phi_{i}\left(A_{j}\right)
$$

This matrix $A_{i j}$ is called the "period matrix". Burnside showed that it is

- (a) real, so $A_{i j}$ gives directly the jump of potential $u_{i}(x, y)$ when going from $C_{j}$ to $C_{j}^{\prime}$

- (b)symmetric: $A_{i j}=A_{j i}$

- (c) positive definite (its eigenvalues are positive).

Before we embark on a discussion of how the period matrix is uniquely defined by the geometry of the problem and is therefore an intrinsic property of the two-dimensional domain under consideration, we want to show how from the knowledge of the $\mathrm{N}$ functions $\phi_{i}(z)$ and the period matrix $A_{i j}$ we can completely solve the problem of the distribution of the function of interest of the system of cylindrical conductors. First we compute the boundary values of $\phi_{i}(z)$ in terms of the period matrix. We make use of a property of the theta series valid in the symmetric case under consideration:

$$
\theta\left(z, J_{i}\right)=-\theta^{*}\left(z^{*}, J_{i}\right)
$$

and then (remember that $z_{0}$ is real)

$$
\phi_{i}\left(z^{*}\right)=-\phi_{i}^{*}(z)
$$

so we find for the real part

$$
u_{i}\left(z^{*}\right)=-u_{i}^{*}(z)
$$

but since $A_{j}^{\prime}=A_{j}$, we find the system of equations

$$
\begin{aligned}
& u_{i}\left(A_{j}^{\prime}\right)+u_{i}\left(A_{j}\right)=0 \\
& u_{i}\left(A_{j}^{\prime}\right)-u_{i}\left(A_{j}\right)=A_{i j}
\end{aligned}
$$

from which we determine the boundary values of $u_{i}(x, y):: u_{i}\left(A_{j}^{\prime}\right)=-u_{i}\left(A_{j}\right)=1 / 2 A_{i j}$

We can now determine the harmonic measures $\omega_{i}(x, y)$ in terms of our set of functions $u_{i}(x, y)$. Since each one of these is the solution of a Dirichlet problem in which the $j$ th circular boundary is set at a value $1 / 2 A_{i j}$, using (30) we can write

$$
u_{i}(x, y)=-\frac{1}{2} \sum_{j=1}^{N} A_{i j} \omega_{j}(x, y)
$$

and inverting it, we find

$$
\omega_{i}(x, y)=-2 \sum_{j=1}^{N} C_{i j} u_{j}(x, y)
$$

where the NxN matrix $C$ with matrix elements $C_{i j}$ is given by the inverse of the period matrix. As the matrix A is positive definite the inverse $A^{-1}$ always exists. The fact that $C=A^{-1}$ together with the last equation solve our problem of determining the harmonic measures in terms of the real part of the function $\phi(z)$ obtained by integrating the Poincare theta series and its associated period matrix $A_{i j}$. We have therefore an explicit algorithm that provides us with an exact solution for the problem under consideration. It is quite interesting to note that the inverse of the period matrix is proportional to the corresponding coefficients of 
the system under consideration (thermal resistance, capacities, etc.). The solution to our problem is given by the potential function

$$
\phi(z)=\sum_{i=1}^{N} \phi_{i} \omega_{i}(x, y)
$$

that finally provides the relationship

$$
q_{i}=-4 \pi \sum_{j=1}^{N} C_{i j} \phi_{j}
$$

Before we end this section we want to make a comment about the period matrix $A_{i j}$. We have defined it as the jump of the function $\phi_{i}(z)$ between a point $A_{j}$ on the boundary $C_{j}$ and its image $A_{j}^{\prime}=T_{j}\left(A_{j}\right)$ on the boundary $C^{\prime}$. However, a fundamental result in the theory of harmonic functions in multiply connected domains states that

$$
A_{i j}=\phi_{i}\left(T_{j}(z)\right)-\phi_{i}(z)
$$

for any point $z$ in the domain under consideration. Using 20 we can then write the period matrix as

$$
A_{i j}=\int_{z}^{T_{j}(z)} \theta\left(z^{\prime}, J_{i}\right) d z^{\prime}=\sum_{\alpha} \ln \left(\frac{T_{\alpha}\left(T_{j}(z)\right)-J_{i}}{T_{\alpha}(z)-J_{i}}\right)
$$

and this should be independent of $z$.

This series of logarithms has the same defect as the series (22) for the $\phi_{i}(z)$ functions, it is an exact solution for the period matrix and the series is convergent, but there are infinite cancelations of logarithms involved. We shall give below an exact series for both $\phi_{i}(z)$ and $A_{i j}$ where this infinite cancellation has already been taken care of. To sum up, in order to solve the problem we are concerned with, one has to compute the period matrix $A_{i j}$ using (35) and the function $\phi_{i}(z)$ using (20). The harmonic measures are then given by (31) and the general solution by (32). Since (35) for $A_{i j}$ does not really depend on the variable $z$ that appears on the right-hand side, it should be clear that the period matrix is a purely geometrical factor depending only on the geometrical configuration of the boundaries involved.

Let us end this section by quoting the improved results for the function $\phi_{i}(z)$ and for the period matrix, once the infinite cancelation is worked out.

$$
\phi_{i}(z)-\phi_{i}\left(z_{0}\right)=\sum_{\alpha \in O_{i}} \ln \left(\frac{z-T_{\alpha}\left(\xi_{i}\right)}{z-T_{\alpha}\left(\eta_{i}\right)} \frac{z_{0}-T_{\alpha}\left(\eta_{i}\right)}{z_{0}-T_{\alpha}\left(\xi_{i}\right)}\right)
$$

with $1=1, \cdots, N$. The sum is restricted to the group elements belonging to the class $O_{i}$ defined as containing the identity $I$ and those elements $T_{\alpha}$ such that does not end up with $T_{i}^{n}$ or $T_{i}^{-n}$. Consequently, the group elements of the form $T_{\beta} T_{i}^{n}$ or $T_{\beta} T_{i}^{-n}$ are not to be included. These are precisely the elements that have been summed up.

The similar improved formula for the period matrix reads

$$
A_{i j}=\delta_{i j} \ln \kappa_{i}+\sum_{\alpha \in O_{i j}} \ln \left(\frac{\xi_{i}-T_{\alpha}\left(\xi_{j}\right)}{\xi_{i}-T_{\alpha}\left(\eta_{j}\right)} \frac{\eta_{i}-T_{\alpha}\left(\eta_{j}\right)}{\eta_{i}-T_{\alpha}\left(\xi_{j}\right)}\right)
$$

Now the sum is restricted to the class $O_{i j}$ of group elements that do not have $T_{j}^{n}$ on the right and $T_{i}^{m}$ on the left $(n, m \neq 0)$. The identity is also excluded because it was explicitly indicated. Notice that this last expression no longer exhibits the spurious dependence on the variable $z$ making evident that $A_{i j}$ only depends on the geometry of the problem.

We comment now on a criterion for the convergence of the series involved in (36) and (37). Remember the definition of the anharmonic ratio of four points, namely,

$$
\begin{aligned}
\left(z_{1}, z_{2}, z_{3}, z_{4}\right) & =\left(z_{1}-z_{2}\right)\left(z_{3}-z_{4}\right) /\left(z_{1}-z_{3}\right)\left(z_{2}-z_{4}\right) \\
& =\left(T\left(z_{1}\right), T\left(z_{2}\right), T\left(z_{3}\right), T\left(z_{4}\right)\right)
\end{aligned}
$$


where he have made explicit the fact that the anharmonic ratio is invariant under any projective transformation of the complex plane onto itself.

In both mentioned series, the argument of the logarithm is an anharmonic ratio. Consequently, we can perform a projective transformation of the type (9) to obtain (for the case (36))

$$
\ln \left(\frac{z^{\prime}-K_{\alpha} \xi_{i}^{\prime}}{z^{\prime}-K_{\alpha} \eta_{i}^{\prime}} \frac{z_{0}^{\prime}-K_{\alpha} \eta_{i}^{\prime}}{z_{0}^{\prime}-K_{\alpha} \xi^{\prime \prime}}\right)
$$

while retaining its value. In this expression, $K_{\alpha}$ is the driving parameter for the convergence of the series. In fact, having defined $\left|K_{\alpha}\right|<1$, one can expand the argument of the logarithm as

$$
1+K_{\alpha}\left(\xi_{i}^{\prime}-\eta_{i}^{\prime}\right)\left(1 / z_{0}^{\prime}-1 / z^{\prime}\right)+O\left(K_{\alpha}^{2}\right)
$$

showing that the rate of convergence of the series is controlled by the smallness of $K_{\alpha}$. The same arguments apply to (37).

\subsection{Green Function}

For some applications of interest, as it is the present case of elasticity, due to the presence of an inhomogeneity it is necessary to solve the Poisson equation. One can treat easily the presence of a source term by knowing the Green function of the domain under consideration. Let us remember that the Green function $G(p, q)$ defined in a multiply-connected domain $D$ bounded by a set of $(M+1)$ circles is the harmonic function of $D$ which vanishes at the boundary and has a logarithmic singularity at $p=q$, namely

$$
G(p, q)=\ln \left|\frac{1}{p-q}\right|+\text { regular terms }
$$

were the regular terms, necessary to ensure the boundary condition, can be explicitly expressed with the aid of the automorphic group technology. In fact, the Green function can be written [6], [9] as

$$
\begin{aligned}
G(p, q)= & \frac{1}{2}\left\{-\sum_{\alpha} \ln \left[\frac{T_{\alpha}(p)-q}{T_{\alpha}(p)-\tilde{q}}\right]+\sum_{\alpha} \ln \left[\frac{T_{\alpha}(\tilde{p})-q}{T_{\alpha}(\tilde{p})-\tilde{q}}\right]\right. \\
& \left.+\sum_{i, j=1}^{M}\left[\phi_{i}(p)-\phi_{i}(\tilde{p})\right]\left(A^{-1}\right)_{i j} \operatorname{Re}\left[\phi_{i}(q)-\phi_{i}(\tilde{q})\right]\right\}
\end{aligned}
$$

where $\tilde{p}$ and $\tilde{q}$ are the inverses of $p$ and $q$ with respect to one particular circle taken as the reference (for example, the external one). Notice that the logarithmic singularity comes from the identity element of the group. In this last expression $\phi_{i}(p)$ is the analytic function given in (36) and $A$ is the period matrix given in (37). From the expression above, and taking into account (21), it is evident that the Green function vanishes on all the boundaries of the domain, as it should be.

Notice that we have been dealing here with the case of Dirichlet boundary conditions but the alternative possibility of Neumann boundary conditions can be equally treated without further difficulties.

\section{Torsion of Bars}

After a brief account of the general problem of torsion of bars of any shape, we particularize our analysis to the case of sections of circular shape and apply all the tools we have introduced in the previous section.

The particular case of bars in which the cylinder has one or several hollows is certainly of practical interest. For this reason, our presentation points to this case that generalizes the typical problem of a cylinder with a solid cross-section and which solution requires extensively the mathematical methods previously presented.

The cross-section of a cylinder of the kind considered is made up of a region $D$ bounded by an external contour $\Gamma_{0}$ and a set of internal contours $\Gamma_{i} ; i=1,2, \cdots, N$. Clearly [1], the torsion function, that we call 
$\Theta(z)$, must satisfy Laplace equation in $D$ with the boundary condition in every contour imposed by the equilibrium condition. As these constraints are not simple to be implemented, it is suitable to introduce the so called stress function $\Psi(z)$ by means of

$$
\begin{aligned}
& \frac{\partial \Theta}{\partial x}=y+2 \frac{\partial \Psi}{\partial y} \\
& \frac{\partial \Theta}{\partial y}=-x-2 \frac{\partial \Psi}{\partial x}
\end{aligned}
$$

This function $\Psi(z)$ satisfies the Poisson equation

$$
\nabla^{2} \Psi(z)=-1
$$

with the property of being $\Psi\left(\Gamma_{0}\right)=0$ while taking constant values on the other contours that are a consequence of the one valuedness, as a function of the coordinates, of the displacement due to the torsion. Consequently, integrating on a closed contour, one has

$$
\oint d \Theta=0=-2 \oint\left(\frac{\partial \Psi}{\partial x} d y-\frac{\partial \Psi}{\partial y} d x\right)-2 \oint(x d y-y d x)
$$

Being the last integral de area inside the integration contour, one can write for each contour

$$
\oint_{\Gamma_{i}} \frac{\partial \Psi(z)}{\partial n_{i}} d \ell=S_{i}
$$

where $S_{i}$ is the area circled by $\Gamma_{i}$.

Just to simplify the presentation of the solution, let us introduce still another auxiliary function $\chi(z)$ such that

$$
\nabla^{2} \chi(z)=-1
$$

with the boundary condition $\chi\left(z \in \Gamma_{j}\right)=0 ; j=0,1,2, \cdots, N$. Taking profit now of the second Green identity, one easily obtains

$$
\chi(z)=\frac{1}{2 \pi} \int_{D} G(z, \zeta) d S_{\zeta}
$$

Just to finally obtain the stress function, one has to include the solution of the homogeneous equation that is written in terms of a set of parameters $\alpha_{i}$ to be fixed in terms of the boundary conditions of $\Psi(z)$, as it was stated before, in order to get a one-valued torsion function. In this way one writes

$$
\Psi(z)=\chi(z)+\sum_{i=1}^{N} \alpha_{i} \omega_{i}(z)
$$

where $\omega_{i}(z)$ are the harmonic measures that, as it was defined before, take the value 1 on the $i$ th boundary and zero on all the others.

In order to determine the parameters $\alpha_{i}$, one uses the condition (46) that in terms of the relation (49) allows to write

$$
S_{i}=\int_{D} d S_{\zeta} \frac{1}{2 \pi} \oint_{\Gamma_{i}} \frac{\partial G(z, \zeta)}{\partial n_{i}} d \ell+\sum_{j=1}^{N} \alpha_{j} \oint_{\Gamma_{i}} \frac{\partial \omega_{j}}{\partial n_{i}} d \ell
$$

Now, the third Green identity provides the relationship

$$
\frac{1}{2 \pi} \oint_{\Gamma_{i}} \frac{\partial G(z, \zeta)}{\partial n_{i}} d \ell=-\omega_{i}(\zeta)
$$

while

$$
\oint_{\Gamma_{i}} \frac{\partial \omega_{j}}{\partial n_{i}} d \ell=A_{i j}
$$


are the elements of the period matrix. Consequently one finds that the parameters $\alpha_{i}$ can be obtained from the expression

$$
\sum_{j=1}^{N} A_{i j} \alpha_{j}=\int_{D} \omega_{i}(\zeta) d S_{\zeta}+S_{i}
$$

where $A_{i j}$ is the period matrix of the domain and $S_{i}$ the area of each hollow.

We end by presenting a closed expression for the factor $K$, torsional rigidity, that measures the stress concentration and is of particular interest in connection with any design of bars subdue to torsion. We give this factor in units of $\mu$, the material dependent modulus of rigidity. Remember that this $K$ factor coincides with the polar moment of inertia of the section when this section is circular. In any other case it is smaller than the polar moment of inertia [10]. Using the definition

$$
K=4 \int_{D} \Psi(z) d S_{z}+4 \sum_{i=1}^{N} \alpha_{i} S_{i}
$$

the final result for the torsional rigidity is

$$
K=\frac{2}{\pi} \iint_{D} G(z, \zeta) d S_{\zeta} d S_{z}+4 \sum_{i, j=1}^{N} \alpha_{i} A_{i j} \alpha_{j}
$$

This expression allows one to compute the $K$ factor for the most general case of a bar section corresponding to a multiply connected domain of circular boundaries.

\subsection{Example}

We consider, as an example of application of the general method just introduced, the case of a cylindrical bar whose cross section is an annulus of internal radius $a$ and external one $b$.

When we introduced the projective transformations and defined the multiplier $\kappa$, we called the attention to the fact that one can always perform a particular projective transformation that maps the invariant points $(\xi, \eta)$ to $(0, \infty)$ by means of the mapping $(9)$. This transformation connects the situation where we are dealing with concentric circles to the one of a given circle an a straight line. In other words, if we were interested in electric problems, this transformation connects the case of coaxial lines with a line over a grounded plane. We present in Fig.(1) the alternatives geometries that we have just described.

With the parameters defined in Fig.(1), the transformation between both complex planes is

$$
z=-\imath \frac{z^{\prime}-b}{z^{\prime}+b}
$$

or equivalently

$$
z^{\prime}=-b \frac{z-\imath}{z+\imath}
$$

and the generator of the group of automorphisms (1), taking into account (13), clearly results in

$$
T(z)=\frac{y_{0} z / R-\imath R\left(y_{0}^{2} / R^{2}-1\right)}{\imath z / R+y_{0} / R}
$$

Notice that in the chosen geometry there is only one generator $T(z)$ and the corresponding invariant points, using (6) result at $\xi=\imath$ and $\eta=-\imath$, respectively. On the other hand, the multiplier (7) results $\kappa=a^{2} / b^{2}$.

In order to build up the Green function, one needs the harmonic measures $\omega_{i}(z)$. Certainly, in the present example there is only one of them. This function is normalized to 1 at the boundary of radius $a$. By using the expression for $\phi(z)$ given in (36), that in the present case includes the only generator $T(z)$, one has

$$
\phi(z)=\ln \left(\frac{z-\xi}{z-\eta}\right)+c t e=\ln \left(\frac{z-\imath}{z+\imath}\right)+c t e
$$




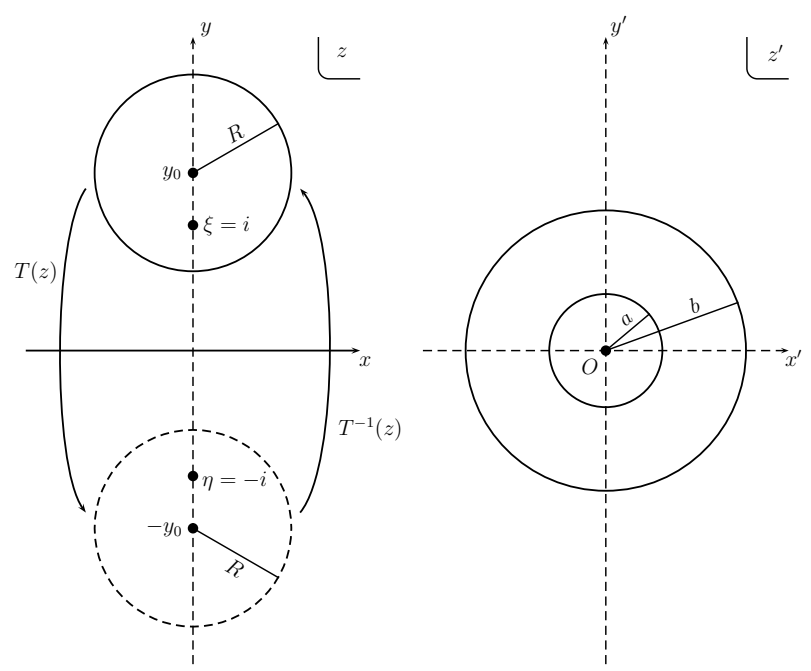

Figure 1: The two different geometries related to the annulus

that, written in terms of $z^{\prime}(55)$ provides

$$
\omega^{\prime}\left(z^{\prime}\right)=\operatorname{Re}\left\{\phi^{\prime}\left(z^{\prime}\right)\right\}=\ln \left(\frac{\mid z^{\prime}}{b}\right)+c t e
$$

that with the appropriate normalization to 1 at the border $a$, reads

$$
\omega^{\prime}\left(z^{\prime}\right)=\frac{\ln \left(\left|z^{\prime}\right| / b\right)}{\ln (a / b)}
$$

It is worth remembering that when only one hole is present, the harmonic measure is directly the real part of the automorphic function. With all the ingredients at our disposal, one can immediately write the Green function of the problem in the $z^{\prime}$-plane

$$
\begin{aligned}
G\left(p^{\prime}, q^{\prime}\right)= & -\ln \left|\frac{p^{\prime}-q^{\prime}}{b-p^{\prime} q^{*} / b}\right|+\frac{\ln \left(\left|p^{\prime}\right| / b\right) \ln \left(\left|q^{\prime}\right| / b\right)}{\ln (a / b)} \\
& -\operatorname{Re}\left\{\sum_{n=-\infty}^{\infty} \ln \left[\frac{b^{2 n} p^{\prime}-a^{2 n} q^{\prime}}{b^{2 n} p^{\prime}-a^{2 n} b^{2} / q^{\prime *}}\right]\right\}
\end{aligned}
$$

This expression shows the singularity at $p^{\prime}=q^{\prime}$ and vanishes at the boundaries, as it should be.

It is possible to give a more adequate form to the Green function by expanding the logarithm in the last term and performing the sum over $n$, the group elements. To this end, it is convenient to introduce polar coordinates, namely

$$
\begin{aligned}
p^{\prime} & =\rho \exp (i \theta) \\
q^{\prime} & =\rho^{\prime} \exp \left(i \theta^{\prime}\right)
\end{aligned}
$$

that verify

$$
a \leq \rho, \rho^{\prime} \leq b
$$

Introducing, as usual, the notation $\rho_{<}$to indicate the smaller of $\rho$ and $\rho^{\prime}$ and $\rho_{>}$to indicate the larger of them and after a little algebra, one ends with

$$
G\left(\rho e^{i \theta}, \rho^{\prime} e^{i \theta^{\prime}}\right)=-\ln \left(\frac{\rho_{<}}{b}\right)+\frac{\ln (\rho / b) \ln \left(\rho^{\prime} / b\right)}{\ln (a / b)}+F\left(\rho, \rho^{\prime}\right) \cos m\left(\theta-\theta^{\prime}\right)
$$


where

$$
F\left(\rho, \rho^{\prime}\right)=\sum_{m=1}^{\infty} \frac{1}{m} \frac{b^{2 m}}{b^{2 m}-a^{2 m}}\left[\left(\frac{\rho_{<}}{\rho_{>}}\right)^{m}+\left(\frac{a^{2} \rho_{>}}{b^{2} \rho_{<}}\right)^{m}-\left(\frac{\rho \rho^{\prime}}{b^{2}}\right)^{m}-\left(\frac{a^{2}}{\rho \rho^{\prime}}\right)^{m}\right]
$$

is the contribution coming from the mentioned expansion of the logarithm in (60). Going back to the expressions (48) and to (10), one gets, after performing the corresponding integrals, the value of the torsion modulus of the annulus

$$
K=\frac{\pi}{2}\left(b^{4}-a^{4}\right)
$$

that certainly coincides with the well known value, given for example in [10].

Clearly, this simple example was included because of its simplicity, just to show how the method is implemented. In the more general cases one has to resort to approximate approaches dictated by the required precision. For example, in the case of a triply connected domain, the automorphic group has now two generators. This fact implies that the series involved in the solution of the problem cannot be summed up directly. One can obtain approximate results by an appropriate selection of the most relevant contributions to the series. In Ref. [4] the triple connected geometry was explicitly considered and an analysis of the convergence of the series was done in terms of the relative distance between the center of the holes and of their radius. It was shown that even in critical configurations, when boundaries are very near to one another, the method provides accurate results.

\section{Final Remarks}

We have adapted the general method for solving the Laplace and the Poisson equations in the framework of projective transformations and the group of automorphism of a surface to the case of torsion of bars.

We call the reader attention to our formula (53) for the torsional rigidity $K$, not only for its completely general character and for being a quite simple tool for performing computations, but also because, up to our present knowledge, it was not stated before.

We have shown, as a trivial example, how our method works in the simple case of a bar whose crosssection is an annulus, obtaining the well known result for the $K$ parameter in this case. The procedure to obtain the torsional rigidity in more general geometries was also discussed.

One can ask oneself if the strong mathematical tool that we have presented is really necessary for obtaining the result of the trivial example above. Obviously, this was only presented in order to see the method doing its job but the important point to be stressed is that this method is absolutely general. It allows one to treat the more complex case of cylindrical symmetry that you can think about. Moreover, it clearly goes beyond the determination of the $K$ factor because it provides the entire solution for the stress function and in this way one can determine the complete distribution of tensions. It is also worth mention that with the same technique, and using the Green identity, one can solve the problem with Neumann boundary conditions to find the torsion function. In this case without going through the function $\chi(z)$.

The general approach we have presented here permits to consider cases where the circular geometrical symmetry is not exact, once the case of the symmetric case is known, by means of the domain variational theory, a mathematical method to systematically treat the general case of boundaries of any shape [11]. This technique allows also the analysis of bars with polygonal cross-sections [12]. We hope to come back to these points in a future publication.

\section{Acknowledgements}

This work was partially supported by ANPCyT, Argentina. We thank S. Sciutto for his kind help in the preparation of the manuscript.

\section{References}

[1] Timoshenko, S.P. and Goodier, J.N., "Theory of Elasticity", 3rd edn. McGraw-Hill, New York 1970. 
[2] Sokolnikoff, I.S., "Mathematical Theory of Elasticity", McGraw-Hill, New York 1946.

[3] Landau, L.D and Lifshitz, E.M., "Theory of Elasticity", Pergamon Press, London 1959.

[4] Alessandrini, V., Fanchiotti, H., García Canal, C.A., Vucetich, H., "Exact Solution for Electrostatic Problem of a System of Parallel Cylindrical Conductors", Journal of Applied Physics, 1974, 45, 3649.

[5] See for example Bird, M.D. and Steele, C.R., "A Solution Procedure for Laplace's Equation on Multiply Connected Circular Domains", Journal of Applied Mechanics, 1992 59, 398.

[6] See for example Schiffer, M and Spencer, D., "Functionals of Finite Riemann Surfaces", Priceton University Press New Jersey 1954; Springer, G. "Introduction to Riemann Surfaces", Addison-Wesley, Reading 1957.

[7] Burnside, W., "On a Class of Automorphic Functions", Proceedings of the London Mathematical Society, 18912349.

[8] See for example: Crowdy D.G. and Marshall J.S., "Green's functions for Laplace's equation in multiply connected domains", IMA. J. Appl. Math, 2007 72, 278; "On the construction of multiply connected quadrature domains", SIAM J. Appl. Math., 2004 64, 1334. D.G. Crowdy, "Explicit solution for the potential flow due to an assembly of stirrers in an inviscid fluid", J. Eng. Math, 2008 62, 333. Y.A. Antipov and V.V. Silvestrov, "Method of automorphic functions in the study of flow around a stack of porous cylinders", Quart. J. Mech. Appl. Math., 2007 60, 337.

[9] Alessandrini, V., "A General Approach to Dual Multiloop Diagrams", Il Nuovo Cimento, 19712321.

[10] Roark, R.J., "Formulas for Stress and Strain", Mc Graw Hill.

[11] See for example: Epele, L.N., Fanchiotti,H., García Canal, C.A., Vucetich, H., "A Domain Variational Theory Approach to the Design of Transmission Line Conductors of Various Cross-Section", International Journal of Electronics, 198456 111;

[12] Epele, L.N., Fanchiotti, H., García Canal, C.A., "Characteristic Impedance of Coaxial Lines Bounded by N Regular Polygons", Proceedings of the IEEE, 198472223. 